\title{
Wine Expertise Predicts Taste Phenotype
}

\author{
John E. Hayes ${ }^{1}$ and Gary J. Pickering ${ }^{2 *}$
}

\begin{abstract}
Taste phenotypes have been studied in relation to alcohol intake, dependence, and family history, with contradictory findings. However, on balance-with appropriate caveats about populations tested, outcomes measured, and psychophysical methods used - an association between variation in taste responsiveness and some alcohol behaviors is supported. Recent work suggests supertasting (operationalized via propylthiouracil [PROP] bitterness) associates not only with heightened response but also with more acute discrimination between stimuli. This work examined relationships between food and beverage adventurousness and taste phenotype. A convenience sample of wine drinkers $(n=331)$ was recruited in Ontario and phenotyped for PROP bitterness via filter paper disk. The subjects also completed a short questionnaire regarding willingness to try new foods, alcoholic beverages, and wines as well as level of wine involvement, which was used to classify each one as a wine expert $(\mathrm{n}=111)$ or a wine consumer $(\mathrm{n}=220)$. In univariate logisitic models, food adventurousness predicted trying new wines and beverages but not expertise. Likewise, wine expertise predicted willingness to try new wines and beverages but not foods. In separate multivariate logistic models, willingness to try new wines and beverages was predicted by expertise and food adventurousness but not PROP. However, mean PROP bitterness was higher among wine experts than wine consumers, and the conditional distribution functions differed between experts and consumers. In contrast, PROP means and distributions did not differ with food adventurousness. These data suggest individuals may self-select for specific professions based on sensory ability (i.e., an active gene-environment correlation), but phenotype does not explain willingness to try new stimuli.
\end{abstract}

Key words: taste genetics, PROP, propylthiouracil, supertasters, food adventurousness, wine adventurousness

The perception of taste and flavor are important factors affecting the liking of food and beverage, and, consequently, purchase decisions. An understanding of individual differences in orosensation is therefore of considerable interest to food and beverage producers, including the wine industry, as these differences may represent important product development and marketing opportunities from market segmentation based on taste types or responsiveness (Pickering and Cullen 2008). Genetic variation is a major determinant of individual differences in orosensation and may be the most important influence on food and beverage behavior (Garcia-Bailo et al. 2009). Responsiveness to the bitterant 6 - $n$-propylthiouracil (PROP) has been widely adopted as a marker of genetic varia-

${ }^{1}$ Department of Food Science, College of Agricultural Sciences, The Pennsylvania State University, University Park, PA; and ${ }^{2}$ Department of Biological Sciences, Cool Climate Oenology and Viticulture Institute, and Department of Psychology, Brock University, St. Catharines, ON L2S 3A1, Canada.

*Corresponding author (email: gary.pickering@brocku.ca; fax: +1-905-6883104)

Acknowledgments: Portions of this work were supported by the Natural Sciences and Engineering Research Council of Canada (Discovery Grant to GJP) and the National Institute of Health grants DC010904 and AA07459 (support for JEH).

The authors thank Lynda Van Zuiden, Valerie Higenell, and Martha Bajec, Brock University, for assistance with data collection; David DiBattista and Gail Higenell, Brock University, and Isabelle Lesschaeve, Vineland Research and Innovation Centre, for advice and assistance; and the study participants for their time and participation.

Manuscript submitted Jun 2011, revised Sept 2011, accepted Oct 2011. Publication costs of this article defrayed in part by page fees.

Copyright (C) 2012 by the American Society for Enology and Viticulture. All rights reserved.

doi: 10.5344/ajev.2011.11050 tion in taste. Individuals have traditionally been classified as PROP nontasters (those for whom PROP elicits no or slight bitterness), PROP medium-tasters (those for whom PROP is mildly bitter), or PROP supertasters (those for whom PROP is intensely bitter). Differential bitterness of PROP associates with variation in the TAS2R 38 gene, but these polymorphisms do not adequately explain supertasting (Hayes et al. 2008).

Importantly, heightened responsiveness to PROP is associated with heightened responsiveness to sensations typically elicited by alcoholic beverages such as burn (Duffy et al. 2004b, Prescott and Swain-Campbell 2000), sourness from carbonation (Prescott et al. 2004), astringency in red wine (Pickering et al. 2004), and bitterness in scotch (Lanier et al. 2005) and beer (Intranuovo and Powers 1998, Lanier et al. 2005) as well as self-reported intake (Duffy et al. 2004a, 2004b), which appears to be mediated via differences in the endogenous sensory properties of these beverages (Lanier et al. 2005). Also, several recent studies show that those who experience PROP as being intensely bitter (i.e., supertasters) not only experience heightened overall oral sensation, but also may be more acute tasters, with the ability to discriminate smaller differences between oral stimuli (Hayes et al. 2010, Lee et al. 2008, Pickering et al. 2004, Prescott et al. 2004).

Personality factors are often ignored or marginalized in taste phenotype research, yet one variable that likely moderates ingestive behaviors is the willingness to try new food and beverages. This willingness varies across individuals and is often conceptualized as food "adventurousness" or "neophobia." Differences in food adventurousness is one possible explanation for contradictory findings on the significance of PROP phenotypes to real world food/beverage preference, liking, and/or intake. For instance, one study speculated that 
previous research may have overestimated the influence of PROP responsiveness on rejection of strong-tasting foods by not distinguishing individuals by food adventurousness (Ullrich et al. 2004). The authors reported that PROP tasters who were more food adventurous liked strong alcohol, hot sauce, chili peppers, other pungent condiments, and bitter fruits and vegetables more than tasters who were less food adventurous. Food neophobia (Logue and Smith 1986) and sensation seeking (Rozin 1990) have previously been linked with different food and/or beverage preferences among individuals. Compared with many other foods/beverages, wine can be viewed as a product for which there is a high level of perceived risk in consumers' minds, given its social cachet, varied nature, and complexity (Lacey et al. 2009). Arguably, this view may attach greater importance to individual adventurousness and "expert" endorsements in mediating consumer preference and purchase decisions.

Purchase decisions for wine consumers are influenced by wine experts or authority figures, particularly wine writers, wine judges, and trained wine retail staff, who help remove some of the perceived risk involved in purchasing wine by providing guidance on quality, taste profile, and relative value to consumers. Thus, an interesting consideration is the extent of concordance in taste phenotype, and particularly PROP, distributions between these wine experts and the typical wine consumers for whom they are making quality judgments and purchase recommendations. A related consideration is that of the taste phenotype distributions of winemakers - a subset of wine experts - relative to wine consumers, given the important role of the winemaker's own palate and attendant taste sensitivities in designing the final product. This research explored the relationship between food and beverage adventurousness and taste phenotype (PROP bitterness) among a cohort of wine drinkers that included both regular consumers and wine experts.

\section{Materials and Methods}

Participants. Individuals of legal drinking age were surveyed at a range of locations around the Niagara Peninsula, which included local Liquor Control Board of Ontario (LCBO) stores (government owned wine and spirits shops in Ontario), staff, faculty, and students at Brock University, Niagara Peninsula wineries, and wine events on and off the Brock campus. The Brock University Ethics Board approved all procedures. Participants were given the opportunity to ask any questions pertaining to the study and their involvement before providing written informed consent. As an incentive, participants were entered in a random drawing for one $\$ 200$ book voucher.

Questionnaire. Participants who reported consuming alcohol beverages were asked to complete a brief questionnaire. Demographic data (age, sex, and ethnicity) were collected, along with food and beverage adventurousness and wine involvement.

Propylthiouracil phenotyping. Propylthiouracil response was determined after the method of Zhao et al. (2003), using filter paper disks impregnated with $50 \mathrm{mmol} / \mathrm{L}$ PROP. Participants placed the disk on their tongues, allowing it to moisten with saliva. They then rated the perceived bitterness of PROP using the generalized labeled magnitude scale (gLMS) (Bartoshuk et al. 2004b). A candy was then provided to participants to help alleviate any lingering bitterness.

Statistical analyses. All analyses were conducted in SAS 9.2 (SAS Institute, Cary, NC). Relationships between trying new wines, beverages, foods, and expertise were compared with univariate and multivariate logistic regression. Group mean bitterness of PROP was compared using $t$-tests, and the distribution of bitter response (kernel density estimates) was compared via proc $k d e$ using the default bandwidth selection options (the Sheather-Jones plug-in [SJPI] method). Here, PROP bitterness was treated as a continuous trait. That is, we did not trichotomize individuals into groups of nontasters, medium-tasters, and supertasters using a priori cutoffs, as such a binning approach is largely a statistical convenience to allow for the use of ANOVA models in analysis. Binning does not reflect the continuous nature of PROP bitterness and costs power (see Hayes and Duffy 2007 for a discussion).

\section{Results}

Participant characteristics. Participants $(n=331)$ were asked to indicate their wine involvement by endorsing given characteristics (Table 1) and were allowed to check as many items as were applicable (259 endorsed one item, 61 endorsed two, 10 endorsed three, and one endorsed four). Among the 51 individuals who indicated they were "another type of wine professional," 11 were amateur winemakers, 10 were winery workers, nine were wine merchants, and five were winery owners. For the present analyses, we classified individuals as wine experts if they were professional winemakers, wine writers, LCBO consultants, wine judges, or some other type of wine professional $(n=111)$. All other participants were classified as nonexperts $(n=220)$.

Food adventurousness, wine expertise, and trying new beverages. Participants indicated how often they try unfamiliar foods and unfamiliar alcohol beverages using a 4-point Likert scale: never, rarely, some of the time, and most of the time. They were asked about their involvement with wine (Table 1) and how often they tried wines that they had not tried before, with the same four response categories. As in other research (Ullrich et al. 2004), we collapsed across response categories to dichotomize individuals into high or

\begin{tabular}{lc}
\hline Table 1 Self-identified characteristics of participants $(\mathrm{n}=331)$. \\
\hline Descriptor [check all that apply] & $\begin{array}{c}\text { Endorsements } \\
(\mathbf{n})\end{array}$ \\
\hline I drink wine only on rare occasions & 14 \\
I drink wine occasionally & 255 \\
I am a professional winemaker & 22 \\
I am a wine writer & 3 \\
I am an LCBO product consultant & 21 \\
I am another type of wine professional & 51 \\
I serve or have served as a judge of commercial & 34 \\
wine at wine show(s) & \\
None of the above & 15 \\
\hline
\end{tabular}


low groups, although we used a different cutoff, collapsing the first three categories in "some of the time or less" for comparison to "most of the time." To maintain consistency, the same cutoff was used across all three questions. For wine adventurousness, 165 individuals were in the low group versus 163 in the high group. For unfamiliar alcohol drinks, 254 individuals were in the low group versus 76 individuals in the high group. For food, the low group had 219 individuals compared to 111 in the high group.

The odds of trying new wines and unfamiliar alcoholic beverages were significantly greater in those individuals who exhibited more food adventurousness (e.g., endorsed trying unfamiliar foods most of the time). Conversely, food adventurousness did not predict wine expertise (Table 2). As expected, the odds of wine experts trying new wines and unfamiliar alcoholic beverages were also greater, and again, no association was seen between wine expertise and willingness to try unfamiliar foods.

Because frequency of trying new wines and unfamiliar alcoholic beverages both associated with frequency of trying new foods, we then tested whether wine experts were more willing to try new wines or drinks when controlling for differences in food adventurousness using multivariate logistic regression (Table 3). In the multivariate analysis, greater food adventurousness and wine expertise made independent contributions to the willingness to try new wines and unfamiliar alcoholic beverages. Propylthiouracil bitterness did not. The relative influence of each predictor differed by outcome measure. Regarding willing to try new wines, wine expertise played a larger role than food adventurousness, whereas the reverse was true for willingness to try new alcoholic drinks (i.e., food adventurousness was more influential than wine expertise).

PROP bitterness, wine expertise, and food adventurousness. When comparing group means, there was a significant effect for PROP bitterness [ $\mathrm{t}(328)=2.16, p=0.03$ ], with experts experiencing higher bitterness than nonexperts. Plots of the bitterness probability distribution functions confirm and extend this finding (Figure 1). For both experts and nonexperts, the smoothed bitterness distributions were trimodal. These three modes are roughly analogous to the nontaster, medium-taster, and supertaster groupings commonly used in the field. Here the same nomenclature is used for comparability with prior work and ease of discussion, while noting that PROP bitterness is a continuous trait and a priori classification cutoffs were not applied here. Notably, the conditional distribution functions indicate wine experts were underrepresented among the nontasters and overrepresented among both the medium- and supertasters. In contrast to the wine expert/wine consumer difference noted above, the mean PROP bitterness did not differ with food adventurousness $[\mathrm{t}(327)=0.26, p=0.8]$.

\section{Discussion}

In this field-based study of adults who consume wine, taste phenotype (PROP bitterness) varied systematically with wine expertise. It has been suggested in a review article that su-

\begin{tabular}{lcccc}
\hline \multicolumn{4}{c}{ Table 3 Summary of multivariate logistic regression. } \\
\hline & Beta & SE & $\begin{array}{c}\text { Odds } \\
\text { ratio }\end{array}$ & $\begin{array}{c}95 \% \\
\text { Wald Cl }\end{array}$ \\
\hline DV: Tries new wines & & & & \\
$\quad$ Wine expert & 1.50 & 0.27 & 4.47 & $2.65-7.54$ \\
Food adventurousness & 0.87 & 0.21 & 2.40 & $1.60-3.61$ \\
PROP bitterness & 0 & 0.01 & 0.99 & $0.99-1.01$ \\
DV: Tries unfamiliar & & & & \\
alcoholic beverages & & & & \\
$\quad$ Wine expert & 1.11 & 0.30 & 3.06 & $1.69-5.54$ \\
Food adventurousness & 1.83 & 0.29 & 6.21 & $3.55-10.86$ \\
PROP bitterness & 0 & 0.01 & 0.99 & $0.99-1.01$ \\
\hline
\end{tabular}

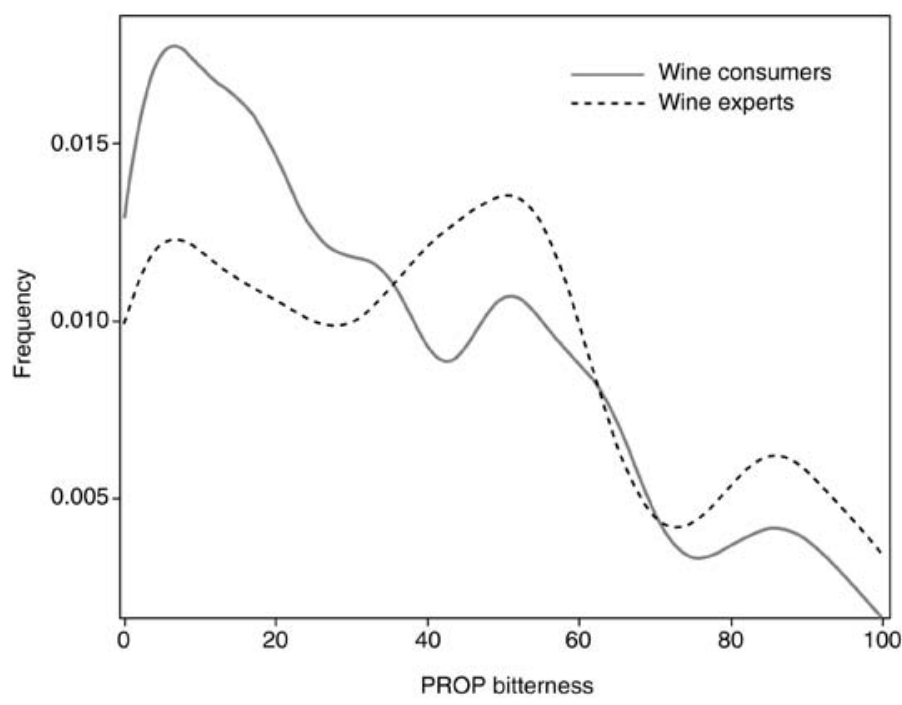

Figure 1 Differences in PROP distribution across wine expertise. The distributions shown are kernel density estimates (KDE) for wine experts and wine consumers (see Materials and Methods and Table 1 for how expertise was determined). Both distributions were trimodal, roughly corresponding to the nontaster, medium-taster, and supertaster groupings classically used in the field.

\begin{tabular}{|c|c|c|c|c|}
\hline Outcome & Predictor & Odds ratio & 95\% Wald Cl & $p$ value \\
\hline Tries new wines & Food adventurousness & 2.29 & $1.56-3.36$ & 0.0001 \\
\hline Tries unfamiliar alcoholic beverages & Food adventurousness & 5.67 & $3.34-9.63$ & 0.0001 \\
\hline Wine expertise & Food adventurousness & 0.84 & $0.58-1.23$ & 0.37 \\
\hline Tries new wines & Wine expert & 4.18 & $2.54-6.90$ & 0.0001 \\
\hline Tries unfamiliar alcoholic beverages & Wine expert & 2.59 & $1.53-4.38$ & 0.0004 \\
\hline Food adventurousness & Wine expert & 1.04 & $0.64-1.69$ & 0.87 \\
\hline
\end{tabular}


pertasters are overrepresented among culinary school students, chefs, and other food experts (Bartoshuk et al. 2004a). Results here show wine experts experienced greater PROP bitterness than wine consumers, and, more interestingly, the distribution of bitterness was skewed toward experts being overrepresented among medium- and supertasters, and underrepresented among nontasters. In addition, food adventurousness and expertise, but not taste phenotype, were related to willingness to try new wines and alcoholic beverages, Finally, the present study treated PROP bitterness as a continuous trait (see Hayes and Duffy 2007 for a discussion), yet even when it was modeled without any binning based on a priori cut-points, the distributions were trimodal, roughly analogous to the non-, medium-, and supertaster labels classically used in the field. A preliminary analysis of the alcoholic beverage liking data collected here suggests a more narrow range of products - almost exclusively wine-define the preference matrix of wine experts than is the case for wine consumers. Future work will examine the possible interaction between PROP responsiveness and wine expertise on alcoholic beverage liking and intake.

Recent work indicates those who taste PROP bitterness more intensely may not only experience heightened intensity across multiple orosensory qualities (e.g., Bajec and Pickering 2008, Hayes et al. 2008) but also have a more acute sense of taste. Specifically, individuals who report greater PROP bitterness show greater discrimination ability within a forced choice paradigm (Prescott et al. 2004), greater changes in intensity and liking across a concentration series (Hayes et al. 2010), and finer discrimination of the mouthfeel qualities elicited by red wine (Pickering and Gordon 2006). Together, these lines of evidence support the idea that individuals may self-select for certain professions (or interests) based on some degree of innate taste advantage. Indeed, we recently found "foodies" (individuals who gave higher affective ratings to food than nonfood items compared to others) were more likely to be supertasters (Minski et al. 2010).

Present work may be initial evidence of an active geneenvironment correlation (rGE) for taste. Within the behavioral genetic taxonomy established elsewhere (Plomin et al. 1977), active rGE occurs when genetically influenced behavior leads an individual to create, seek, or select an environment that matches their genotype (see Rutter and Silberg 2002), thus enhancing expression of a phenotype. Thus, we postulate that individuals who have greater native ability to discriminate between foods and beverages preferentially select for professions (chefs, wine experts) where this enhanced ability provides some competitive advantage. Superior innate ability would not, in and of itself, constitute a substitute for expertise developed over time, as an individual would also need to first have the desire to learn about wine and then to develop their ability to communicate those sensations to others: that is, the shift in the basic-object level (see Solomon 1991) from describing a wine as a dry versus sweet white wine to describing it as a Kabinett versus a Trockenbeerenauslese Riesling depends on learning, not physiology. Still, one study reported that PROP supertasters use more terms to describe dairy products in free-choice profiling (Kirkmeyer and Tepper 2003), consistent with the idea that the language of experts may reflect a more nuanced appreciation of the physical world (Solomon 1990). However, additional data is needed to confirm the rGE hypothesis.

The above speculation presupposes that perception of the orosensory sensations elicited by wine does, in fact, vary with PROP responsiveness. In studies that have used the accepted suprathreshold methods (as opposed to threshold-based phenotyping methods) to characterize individuals, this proposition is supported by the findings of Pickering et al. (2004) and Pickering and Gordon (2006). Similarly, the sensations elicited from beer and blended scotch whisky also associate with PROP responsiveness (Lanier et al. 2005). Pickering et al. (2010b) failed to find an association between PROP responsiveness and sensations elicited by white and red wines. However, this null result may be due to the use of pectin as an interstimulus rinse, as pectin can bind proteins and polymorphisms in the salivary protein gustin may account for the different taste responsiveness between PROP phenotypes (Padiglia et al. 2010). Also, it is important to note that all 330 individuals in the present study were already wine drinkers, regardless of level of expertise. Thus, present data do not contradict the taste phenotype alcohol protection hypothesis found in the addiction and psychophysics literature. That is, there may be plenty of individuals in the population who experience heightened PROP bitterness and never acquire a taste for alcohol (the "tastes too strong" protection hypothesis), but because our sample was recruited at wine events, those individuals would not be included in the data set. Instead, our data indicate that heightened bitterness and taste acuity may encourage people to become wine experts once they are already wine consumers. Clearly, learning and opportunity also play a role in this process.

Regarding willingness to try new wines, expertise was more important than food adventurousness, while the reverse was true for willingness to try new alcoholic drinks. This finding makes intuitive sense, as experts must try new wines for professional reasons, whereas willingness to try other beverages may be more similar to their typical eating behavior. In contrast, mean bitterness did not differ with food adventurousness, nor was PROP response significant in the multivariate logistic regression models, suggesting that taste phenotype does not influence willingness to try new foods, drinks, or wines, at least in this sample.

\section{Conclusion}

The finding that wine experts are more likely to be medium-tasters or supertasters than other wine consumers may suggest a possible discordance in judgments of quality and value between the two groups. Wine consumers may wish to apply additional caution in adopting wine expert endorsements or recommendations. Assessment of wine quality is dependent on both experience (and resulting expectancies) and liking, which is associated with taste responsiveness; both of these appear to vary between wine authority figures and wine consumers. 


\section{Literature Cited}

Bajec, M.R., and G.J. Pickering. 2008. Thermal taste, PROP responsiveness, and perception of oral sensations. Physiol. Behav. 95(4):581-590.

Bartoshuk, L.M, V.B. Duffy, A.K. Chapo, K. Fast, J.H. Yiee, H.J. Hoffman, C.W. Ko, and D.J. Snyder. 2004a. From psychophysics to the clinic: Missteps and advances. Food. Qual. Pref. 15(7-8):617-632.

Bartoshuk, L.M, V.B. Duffy, B.G. Green, H.J. Hoffman, C.W. Ko, L.A. Lucchina, L.E. Marks, D.J. Snyder, and J.M. Weiffenbach. 2004b. Valid across-group comparisons with labeled scales: The gLMS versus magnitude matching. Physiol. Behav. 82(1):109-114.

Duffy, V.B., A.C. Davidson, J.R. Kidd, K.K. Kidd, W.C. Speed, A.J. Pakstis, D.R. Reed, D.J. Snyder, and L.M. Bartoshuk. 2004a. Bitter receptor gene (TAS2R38), 6- $n$-propylthiouracil (PROP) bitterness and alcohol intake. Alcohol Clin. Exp. Res. 28(11):1629-1637.

Duffy, V.B., J. Peterson, and L.M. Bartoshuk. 2004b. Associations between taste genetics, oral sensations and alcohol intake. Physiol. Behav. 82(2-3):435-445.

Garcia-Bailo, B., C. Toguri, K.M. Eny, and A. El-Sohemy. 2009. Genetic variation in taste and its influence on food selection. Omics 13:69-80.

Hayes, J.E., and V.B. Duffy. 2007. Revisiting sugar-fat mixtures: Sweetness and creaminess vary with phenotypic markers of oral sensation. Chem. Senses 32(3):225-236.

Hayes, J.E., L.M. Bartoshuk, J.K. Kidd, and V.B. Duffy. 2008. Supertasting and PROP bitterness depends on more than the TAS 2 R 38 gene. Chem. Senses 33(3):255-265.

Hayes, J.E., B.S. Sullivan, and V.B. Duffy. 2010. Explaining variability in sodium intake through oral sensory phenotype, salt sensation and liking. Physiol. Behav. 100(4):369-380.

Intranuovo, L.R., and A.S. Powers. 1998. The perceived bitterness of beer and 6-n-propylthiouracil (PROP) taste sensitivity. Ann. N.Y. Acad. Sci. 855:813-815.

Kirkmeyer, S.V., and B.J. Tepper. 2003. Understanding creaminess perception of dairy products using free-choice profiling and genetic responsivity to 6- $n$-propylthiouracil. Chem. Senses 28(6):527-536.

Lacey, S., J. Bruwer, and E. Li. 2009. The role of perceived risk in wine purchase decisions in restaurants. Int. J. Wine Bus. Res. 21(2):99-117.

Lanier, S.A., J.E. Hayes, and V.B. Duffy. 2005. Sweet and bitter tastes of alcoholic beverages mediate alcohol intake in of-age undergraduates. Physiol. Behav. 83(5):821-831.

Lee, Y.M., J. Prescott, and K.O. Kim. 2008. PROP taster status and the rejection of foods with added tastants. Food Sci. Biotechnol. 17(5):1066-1073.

Logue, A.W., and M.E. Smith. 1986. Predictors of food preferences in adult humans. Appetite 7(2):109-125.

Minski, K.R., L.M. Bartoshuk, J.E. Hayes, H.J. Hoffman, S. Rawal, and V.B. Duffy. 2010. NIH Toolbox: Proposed food liking survey. Abst. Chem. Senses 35(7):A21.
Padiglia, A, A. Zonza, E. Atzori, C. Chillotti, C. Calo, B.J. Tepper, and I.T. Barbarossa. 2010. Sensitivity to 6- $n$-propylthiouracil is associated with gustin (carbonic anhydrase VI) gene polymorphism, salivary zinc, and body mass index in humans. Am. J. Clin. Nutr. 92(3):539-545.

Pickering, G.J., and C.W. Cullen. 2008. The influence of taste sensitivity and adventurousness on Generation Y's liking scores for sparkling wine. In Proceedings of the Fourth International Conference of the Academy of Wine Business Research. Siena, Italy, 17-19 July 2008 (CD).

Pickering, G.J., and R. Gordon. 2006. Perception of mouthfeel sensations elicited by red wine are associated with sensitivity to 6- $n$ propylthiouracil. J. Sens. Stud. 21:249-265.

Pickering, G.J., J.A. Bartolini, and M.R. Bajec. 2010a. Perception of beer flavour associates with thermal taster status. J. Inst. Brew. 116(3):239-244

Pickering, G.J., A. Moyes, M.R. Bajec, and N. Decourville. 2010b. Thermal taster status associates with oral sensations elicited by wine. Aust. J. Grape Wine Res. 16(2):361-367.

Pickering, G.J., K. Simunkova, and D. DiBattista. 2004. Intensity of taste and astringency sensations elicited by red wines is associated with sensitivity to PROP (6-n-propylthiouracil). Food. Qual. Pref. 15(2):147-154

Plomin, R., J.C. DeFries, and J.C. Loehlin. 1977. Genotype-environment interaction and correlation in the analysis of human behavior. Psychol. Bull. 84:309-322.

Prescott, J., and N. Swain-Campbell. 2000. Responses to repeated oral irritation by capsaicin, cinnamaldehyde and ethanol in PROP tasters and non-tasters. Chem. Senses 25(3):239-246.

Prescott, J., J. Soo, H. Campbell, and C. Roberts. 2004. Responses of PROP taster groups to variations in sensory qualities within foods and beverages. Physiol. Behav. 82(2-3):459-469.

Rozin, P. 1990. Getting to like the burn of chili pepper: Biological, psychological and cultural perspectives. In Chemical Senses. Vol. 2. Irritation. B.G. Green et al. (eds.), pp. 231-269. Marcel Dekker, New York.

Rutter, M., and J. Silberg. 2002. Gene-environment interplay in relation to emotional and behavioral disturbance. Ann. Rev. Psych. 53:463-490.

Solomon, G.E. 1990. Psychology of novice and expert wine talk. Am. J. Psych. 103(4):495-517.

Solomon, G.E.A. 1991. Language and categorization in wine expertise. In Sensory Science: Theory and Applications in Foods. H.T. Lawless and B.P. Klein (eds.), pp. 269-294. Marcel Dekker, New York.

Ullrich, N.V., R. Touger-Decker, J. O'Sullivan-Maillet, and B.J. Tepper. 2004. PROP taster status and self-perceived food adventurousness influence food preferences. J. Am. Diet. Assoc. 104(4):543-549.

Zhao, L., S.V. Kirkmeyer, and B.J. Tepper. 2003. A paper screening test to assess genetic taste sensitivity to $6-n$-propylthiouracil. Physiol. Behav. 78(4-5):625-633 